\title{
Asymptotic Behavior of a Tumor Angiogenesis Model with Haptotaxis
}

\section{Chi $\mathrm{Xu} *$ and Yifu Wang}

School of Mathematics and Statistics, Beijing Institute of Technology, Beijing 100081, China; wangyifu@bit.edu.cn

* Correspondence: XuChi1993@126.com

Received: 10 February 2020; Accepted: 15 March 2020; Published: 27 April 2020

Abstract: This paper considers the existence and asymptotic behavior of solutions to the angiogenesis system $p_{t}=\Delta p-\rho \nabla \cdot(p \nabla w)+\lambda p(1-p), w_{t}=-\gamma p w^{\beta}$ in a bounded smooth domain $\Omega \subset$ $\mathbb{R}^{N}(N=1,2)$, where $\rho, \lambda, \gamma>0$ and $\beta \geq 1$. More precisely, it is shown that the corresponding solution $(p, w)$ converges to $(1,0)$ with an explicit exponential rate if $\beta=1$, and polynomial rate if $\beta>1$ as $t \rightarrow \infty$, respectively, in $L^{\infty}$-norm.

Keywords: angiogenesis; haptotaxis; asymptotic behavior

AMS Subject Classification: 35B40; 35K57; 35B65; 92C17; 35Q92

\section{Introduction}

As a physiological process, angiogenesis involves the formation of a new capillary network sprouting from a pre-existing vascular network. It has been recognized that the capillary growth through angiogenesis leads to vascularization of tumor, providing it with its own blood supply. During this process, the endothelial cells are induced by the fibronectin, which are bounded in Extracellular Matrix (ECM) and gathered under the effect of fibronection to form new vessels. In [1], Anderson et al. proposed a reaction-diffusion model to describe those procedures. In [2], Stevens and Othmer developed the so-called reinforced random walk to gain the understanding of the mechanism that causes the aggregation of myxobacteria. By the methodology established in [2], Levine et al. [3,4] derived models of the angiogenesis based on analysis of the relevant biochemical processes. We refer to $[5,6]$ for the related research.

In this paper, we are concerned with the initial-boundary problem

$$
\begin{cases}p_{t}=\Delta p-\rho \nabla \cdot(p \chi(w) \nabla w)+f(p, w), & x \in \Omega, t>0 \\ w_{t}=-\gamma p w^{\beta}, & x \in \Omega, t>0 \\ \frac{\partial p}{\partial v}-p \rho \frac{\partial w}{\partial v}=0, & x \in \partial \Omega, t>0 \\ u(x, 0)=u_{0}(x), w(x, 0)=w_{0}(x), & x \in \partial \Omega, t>0\end{cases}
$$

Here $\Omega \subset \mathbb{R}^{N}(N=1,2)$ is a bounded domain with smooth boundary $\partial \Omega$ and $v$ is unit outward normal vector. $p$ denotes the density of endothelial cells and $w$ represents the density of concentration of chemical substance such as fibronectin; $\rho$ and $\gamma$ are positive constant; $\beta \geq 1$. $f(p, w)$ denotes proliferation of endothelial cells; and $\chi(w)$ is called chemosensitivity. In addition to random motion, endothelial cells have haptotaxis migration, which responds to the gradient of attractant such as the fibronectin that is non-diffusible in the extracellular matrix.

Mathematical models with haptotaxis have attracted lots of attention [7-12]. For example, Corrias et al. [7] considered the system in Equation (1) with $f \equiv 0$ and $\beta=1$ in the bounded domain and whole space, respectively. In fact, they proved that there exists a global-in-time 
$L^{1}$-bounded weak solution when the domain is bounded and the sensitivity function $\chi(w)>0$, $\inf _{w \geq 0} \frac{w \chi^{\prime}(w)}{\chi(w)}>-1$. Moreover, the solution $p$ converges to $\frac{1}{|\Omega|} \int_{\Omega} p_{0}$ in $L^{1}(\Omega)$ weakly and $c$ decays to zero in $L^{p}(\Omega)(p<\infty)$ in strong topology. For the unbounded domain and the initial data satisfying $p_{0} \log \left(1+|x|^{N+1}\right) \in L^{1}\left(\mathbb{R}^{N}\right)$, they obtained the existence of weak solution and the self-similar solutions thereof. Furthermore, they extended their previous result to the case of $\beta \geq 1$ in [8]. Guarguaglini et al. [9] considered the variant of Equation (1) with $\chi(w)=\frac{1}{a+b w}, f \equiv 0$, and $w$-equation

$$
\frac{d}{d t} w=-p(a+b w) w
$$

in whole line. Under some suitable assumption, it is shown that the corresponding system admits a global weak solution and local classic solution for sufficiently regular initial data. On the other hand, Rascle [12] showed the local existence and uniqueness of classic solutions of the system in Equation (1) with boundary condition $\frac{\partial p}{\partial v}=0$ instead of $\frac{\partial p}{\partial v}-p \rho \frac{\partial w}{\partial v}=0$ where $w(\cdot, 0) \equiv w_{0}>0$ is a positive constant and particularly

$$
|f(p, w)| \leq L|p|
$$

with some $L>0$ for all $p \geq 0, w \geq 0$. Liţcanu and Morales-Rodrigo [10] studied the system with $\chi(w) \equiv 1$, and standard logistic growing source. Indeed, they proved the system in Equation (1) admits a globally $L^{\infty}$-bounded classic solution which converges to a constant with polynomial rate when $\beta>1$ and exponential rate if $\beta=1$ in $L^{2}$-norm, respectively, when the initial data have a positive lower bound.

It is noted that modeling approaches indicate that, in situations of significantly heterogeneous environment, adequate macroscopic limits of random walk rather lead to certain non-Fickian diffusion operator. We refer to $[13,14]$ for the fractional diffusion and refer to $[15,16]$ for the nonlinear diffusion. It is should be mentioned that Winkler [17] considered the related haptotaxis system of Equation (1), which describes the glioma spread in heterogeneous tissue, and proved the system has global weak solution with few initial data and the solution component $p$ stabilizes towards a state involving infinite densities and other component $w$ tends to zero. Finally, we would like mention some papers [18,19] where $w$ satisfies

$$
\frac{d}{d t} w=g(p, w)
$$

and assumptions on $g$ are much more restricted.

Our aim is to consider the system in Equation (1) with $\chi(w) \equiv 1$ and a standard logistic growing source, namely

$$
\begin{cases}p_{t}=\Delta p-\rho \nabla \cdot(p \nabla w)+\lambda p(1-p), & x \in \Omega, t>0, \\ w_{t}=-\gamma p w^{\beta}, & x \in \Omega, t>0, \\ \frac{\partial p}{\partial v}-p \rho \frac{\partial w}{\partial v}=0, & x \in \partial \Omega, t>0 \\ p(x, 0)=p_{0}(x), w(x, 0)=w_{0}(x) & x \in \partial \Omega, t>0 .\end{cases}
$$

We assume the initial data satisfy

$$
\left\{\begin{array}{l}
\text { where }\left(p_{0}, w_{0}\right) \in C^{2+\alpha}(\bar{\Omega})^{2} \text { is nonnegative for some } \alpha \in(0,1) \text { with } p_{0} \not \equiv 0 \\
\frac{\partial p_{0}}{\partial v}-p_{0} \rho \frac{\partial w_{0}}{\partial v}=0 \text { on } \partial \Omega .
\end{array}\right.
$$

The main result can be stated as follows:

Theorem 1. Let $\gamma \lambda, \rho$ be positive parameter and $\beta \geq 1$. Then the problem in Equations (2) and (3) admits a globally $L^{\infty}$-bounded positive classic solution which satisfies

$$
\lim _{t \rightarrow \infty}\|p(\cdot, t)-1\|_{L^{\infty}(\Omega)}+\|w(\cdot, t)\|_{L^{\infty}(\Omega)}=0 .
$$


Moreover,

(1) If $\beta=1$, then for any $\epsilon \in\left(0, \min \left\{\lambda_{1}, 1, \gamma, \lambda\right\}\right)$, there exists $C(\epsilon)>0$ such that:

$$
\begin{gathered}
\|p(\cdot, t)-1\|_{L^{\infty}(\Omega)} \leq C(\epsilon) e^{-\left(\min \left\{\lambda_{1}, 1, \gamma, \lambda\right\}-\epsilon\right) t}, \\
\|w(\cdot, t)\|_{L^{\infty}(\Omega)} \leq C(\epsilon) e^{-\gamma(1-\epsilon) t},
\end{gathered}
$$

where $\lambda_{1}$ is the first nonzero eigenvalue of $-\Delta$ in $\Omega$ with the homogeneous Neumann boundary condition.

(2) If $\beta>1$, then for any $\epsilon \in(0,1)$, there exist a constant $C>0$ and $t_{\epsilon}$ such that

$$
\begin{gathered}
\|p(\cdot, t)-1\|_{L^{\infty}(\Omega)} \leq C(\epsilon)\left(\gamma(\beta-1)(1-\epsilon)\left(t-t_{\epsilon}\right)+\left\|w_{0}\right\|_{L^{\infty}(\Omega)}^{1-\beta}\right)^{\frac{1}{2(1-\beta)}}, \\
\|w(\cdot, t)\|_{L^{\infty}(\Omega)} \leq C\left(\gamma(\beta-1)(1-\epsilon)\left(t-t_{\epsilon}\right)+\left\|w_{0}\right\|_{L^{\infty}(\Omega)}^{1-\beta}\right)^{\frac{1}{1-\beta}} .
\end{gathered}
$$

for all $t>t_{\epsilon}$

Remark 1. The system under consideration is very similar to the problem in [10]. However, we remove the condition that the initial data must have a positive lower bound to reach the $L^{\infty}$-convergence of solution.

The crucial idea towards to the proof of Theorem 1 in our approach is to derive a bound for $\int_{\Omega} q^{2^{m+1}}+\int_{t}^{t+\tau} \int_{\Omega}\left|\nabla q^{2^{m}}\right|^{2}$ from the bound of $\int_{t}^{t+\tau} \int_{\Omega} q^{2^{m}}(m=1,2 \cdots)$ with some constant $\tau<1$ and the inequality

$$
\frac{d}{d t} F(p, w) \leq-\int_{\Omega} \frac{|\nabla p|^{2}}{p}-C \int_{\Omega} p|\nabla w|^{2}
$$

with

$$
F(p, w)=\frac{\rho}{2 \gamma} \int_{\Omega} w^{-\beta}|\nabla w|^{2}+\int_{\Omega} p(\log p-1) .
$$

for some $C>0$. Furthermore, with the help of estimate of $q=p e^{-p w}$ and Gagliardo-Nirenberg interpolation theorem, we can show the component $p(x, t)$ converges to 1 in $L^{\infty}(\Omega)$ as $t \rightarrow \infty$ with exponential rate if $\beta=1$ and polynomial rate in $L^{\infty}$-norm if $\beta>1$, respectively. This paper is organized as follows. In Section 2 , we prove the system exists a globally $L^{\infty}$-bounded classic solution for any $\beta \geq 1$ by the iterated method. In Section 3, we show the solution converge to stationary solution in $L^{\infty}$-norm when $N=2$ and establish the explicit decay rate of solution in case of $\beta>1$ and $\beta=1$, respectively. In Section 4, we prove the same result in one-dimensional setting.

\section{Global Boundedness of Solution}

\subsection{Local Existence}

In this section, we state the local existence and uniqueness of solution to problem in Equations (2) and (3).

Lemma 1. ([20]) Let $\Omega \subset \mathbb{R}^{N}$ be a smooth bounded domain. If the initial data satisfy Equation (3), then there exists $T_{\max }<\infty$ such that the problem in Equations (2) and (3) admits a unique positive classic solution $(p, w) \in\left(C^{2+\alpha, 1+\frac{\alpha}{2}}\left(\bar{\Omega} \times\left[0, T_{\max }\right]\right)\right)^{2}$ with $\alpha=1-\frac{N+2}{s}$ and $s>N+2$. Moreover,

$$
\lim _{t \rightarrow \infty} \sup \|p(\cdot, t)\|_{W^{1, s}(\Omega)} \rightarrow+\infty
$$

if $T_{\max }<+\infty$.

Proof. We refer to Theorems 2.1 and 2.2 of [20] for the details of proof thereof. 


\subsection{Global Existence}

This section is devoted to the global existence of classic solution to problem in Equations (2) and (3). To this end, we derive some prior estimates of $(p, w)$ and henceforth fix $\tau=\min \left\{1, \frac{T_{\max }}{6}\right\}$.

Lemma 2. ([20]) For all $t<T_{\max }$

$$
\begin{gathered}
0 \leq \int_{\Omega} p \leq C_{1}:=\max \left\{\int_{\Omega} p_{0},|\Omega|\right\} \\
\int_{\tau}^{t+\tau} \int_{\Omega} p^{2} \leq C_{2}:=C_{1}\left(\frac{1}{\lambda}+1\right) \text { for all } t \in\left(0, T_{\max }-\tau\right) . \\
\left|\int_{0}^{t} \int_{\Omega} p(1-p)\right| \leq \frac{C_{1}}{\lambda} \text { for all } t \in\left(0, T_{\max }\right) .
\end{gathered}
$$

Lemma 3. We have following estimates

$$
|w(\cdot, t)| \leq \|\left. w_{0}\right|_{L^{\infty}(\Omega)}
$$

for all $t \leq T_{\max }$.

Proof. By solving $w$-equation in Equation (2), we obtain

$$
w(x, t)=\left((\beta-1) \gamma \int_{0}^{t} p(s) d s+w_{0}^{1-\beta}\right)^{\frac{1}{1-\beta}} .
$$

Therefore, the positivity of $p$ implies that

$$
|w(x, t)| \leq\left\|w_{0}\right\|_{L^{\infty}(\Omega)} .
$$

Lemma 4. For any $t \leq T_{\max }$ and $n \in(1, \infty)$, there exists constant $C(n, \tau)>0$ such that

$$
\|p(\cdot, t)\|_{L^{n}(\Omega)} \leq C
$$

Proof. Let $q=e^{-\rho w} p$. Then, $p=q e^{\rho w}$, which satisfies

$$
q_{t} e^{\rho w}=\operatorname{div}\left(e^{\rho w} \nabla q\right)+\lambda e^{\rho w} q\left(1-e^{\rho w} q\right)-\rho \gamma q^{2} e^{2 \rho w} w^{\beta} .
$$

It is observed that

$$
\begin{aligned}
\frac{1}{n} \frac{d}{d t}\left(q^{n} e^{\rho w}\right) & =q^{n-1}\left(\operatorname{div}\left(e^{\rho w} \nabla q\right)+\lambda e^{\rho w} q\left(1-e^{\rho w} q\right)-\rho \gamma q^{2} e^{2 \rho w} w^{\beta}\right)+\frac{1}{n} \rho \gamma q^{n+1} e^{\rho w} w^{\beta} \\
& =q^{n-1} \operatorname{div}\left(e^{\rho w} \nabla q\right)+\lambda e^{\rho w} q^{n}\left(1-e^{\rho w} q\right)+\frac{1-n}{n}\left(\gamma \rho q^{n+1} e^{2 \rho w} w^{\beta}\right)
\end{aligned}
$$

with $n \geq 2$. Integrating the above equality over $\Omega$ yields

$$
\frac{d}{d t} \int_{\Omega} q^{n} e^{\rho w}+2 \int_{\Omega}\left|\nabla q^{\frac{n}{2}}\right|^{2} \leq k_{0} \int_{\Omega} q^{n}+\left(k_{1}-\lambda\right) \int_{\Omega} q^{n+1},
$$

where $k_{0}=k_{0}\left(\lambda, \rho, \beta,\left\|w_{0}\right\|_{L^{\infty}(\Omega)}\right), k_{1}=k_{1}\left(n, \rho, \gamma, \beta,\left\|w_{0}\right\|_{L^{\infty}(\Omega)}\right)$. We set $z=q^{\frac{n}{2}}$ and then

$$
\frac{d}{d t} \int_{\Omega} z^{2} e^{\rho w}+2 \int_{\Omega}|\nabla z|^{2} \leq n k_{0} \int_{\Omega} z^{2}+n\left(k_{1}-\lambda\right) \int_{\Omega} z^{2+\frac{2}{n}}
$$


Without loss of generality, we assume $z>1$ and apply the Gagliardo-Nirenberg inequality and Cauchy inequality yields

$$
\begin{aligned}
\int_{\Omega} z^{2+\frac{2}{n}} & \leq C\|z\|_{H^{1}(\Omega)}|| z \mid \|_{L^{1+\frac{2}{n}}(\Omega)}^{1+\frac{2}{n}} \\
& \leq C\|z\|_{H^{1}(\Omega)}\|z\|_{L^{2}(\Omega)}^{1+\frac{2}{n}} \\
& \leq \epsilon\|z\|_{H^{1}(\Omega)}^{2}+\frac{C}{\epsilon}\|z\|_{L^{2}(\Omega)}^{4}+1 .
\end{aligned}
$$

Hence, Equation (16) can be rewritten as:

$$
\begin{aligned}
& \frac{d}{d t} \int_{\Omega} z^{2} e^{\rho w}+\left(2-n\left(k_{1}-\lambda\right) \epsilon\right) \int_{\Omega}|\nabla z|^{2} \\
& \leq\left(n k_{0}+n\left(k_{1}-\lambda\right) \epsilon\right)\|z\|_{L^{2}(\Omega)}^{2}+\frac{n\left(k_{1}-\lambda\right) C}{\epsilon}\|z\|_{L^{2}(\Omega)}^{4}+C
\end{aligned}
$$

We take $\epsilon$ small enough such that $\left(2-n\left(k_{1}-\lambda\right) \epsilon\right)>1$ to obtain that

$$
\frac{d}{d t} \int_{\Omega} z^{2} e^{\rho w}+\int_{\Omega}|\nabla z|^{2} \leq c_{1}|| z\left\|_{L^{2}(\Omega)}^{2}+c_{2}|| z\right\|_{L^{2}(\Omega)}^{4}+C
$$

where $c_{1}=n k_{0}+n\left(k_{1}-\lambda\right) \epsilon$ and $c_{2}=\frac{n\left(k_{1}-\lambda\right)}{C}$. Now, we define $y_{m}=\int_{\Omega} q^{2^{m}} e^{\rho w}$ and $z_{m}=q^{2^{m}}$. Then, we have

$$
\frac{d}{d t} y_{m}+\int_{\Omega}\left|\nabla z_{m-1}\right|^{2} \leq b(t) y_{m}+C
$$

where

$$
b(t)=c_{1}+c_{2} \int_{\Omega} z_{m} .
$$

To complete the proof of this lemma, it suffices to show that

$$
\int_{\Omega} q^{2^{m}} e^{\rho w}+\int_{t}^{t+\tau} \int_{\Omega}\left|\nabla q^{2^{m-1}}\right|^{2} \leq C(m)
$$

is valid for any integer $m \geq 1$. We mention here the constant $C$ is different from that in Equation (17). In the following, we use induction to prove Equation (19).

For $m=1$ and any given $t>\tau$, we can find some $t_{0} \in[t-\tau, t]$ such that $y_{1}\left(t_{0}\right)=\frac{C_{1}}{\tau}\left(1+\frac{1}{\lambda}\right)$ if $t>\tau$ and $t_{0}=0$ if $t \leq \tau$. By solving Equation (18), we have

$$
y_{1}(t) \leq y_{1}\left(t_{0}\right) e^{\int_{t_{0}}^{t} b(s) d s}+c_{3} \int_{t_{0}}^{t} e^{\int_{s}^{t} b(\sigma) d \sigma} d s<c_{4} .
$$

In the above inequality, the fact $\int_{t-\tau}^{t} b(s) d s \leq C$ has been used. Now, we assume

$$
\int_{\Omega} q^{2^{k-1}} e^{\rho w}+\int_{t}^{t+\tau} \int_{\Omega}\left|\nabla q^{2^{k-2}}\right|^{2} \leq C(k-1)
$$

is valid for $m=k-1 \geq 1$. Due to the Gagliardo-Nirenberg inequality,

$$
\|z\|_{L^{4}(\Omega)}^{4} \leq c_{4}\|\nabla z\|_{L^{2}(\Omega)}^{2}|| z\left\|_{L^{2}(\Omega)}^{2}+c_{5}\right\| z \|_{L^{2}(\Omega)}^{4}
$$

which implies

$$
\int_{\Omega} q^{2^{k}} \leq c_{4} \int_{\Omega}\left|\nabla q^{2^{k-2}}\right|^{2} \int_{\Omega} q^{2^{k-1}}+c_{5}\left(\int_{\Omega} q^{2^{k-1}}\right)^{2}
$$


It follows that

$$
\int_{t}^{t+\tau} \int_{\Omega} q^{2^{k}} \leq C
$$

and thereby

$$
\int_{\Omega} q^{2^{k}}\left(t_{0}\right) \leq \frac{C}{\tau}
$$

for some $t_{0} \in[t-\tau, t]$. By the inequality

$$
\frac{d}{d t} y_{k}+\int_{\Omega}\left|\nabla q^{2^{k-1}}\right|^{2} \leq b(t) y_{k}+C
$$

we have

$$
\int_{\Omega} q^{2^{k}} e^{\rho w}+\int_{t}^{t+\tau} \int_{\Omega}\left|\nabla q^{2^{k-1}}\right|^{2} \leq C(k) .
$$

With the help of induction, one can see that Equation (19) is valid for all $m \geq 1$. The argument above is still valid in one-dimension setting with some adaption.

Lemma 5. There exists a constant $C$ such that

$$
\|p(\cdot, t)\|_{L^{\infty}(\Omega)}<C .
$$

for all $t \in\left(0, T_{\max }\right)$.

Proof. Now, we define $q_{k}=(q-k)_{+}$, where $(\cdot)_{+}$denotes the positive part of function and $\Omega_{k}(t)=$ $\{x \in \Omega: q(x, t)>k\}$. Then, it is observed that

$$
\frac{1}{2} \frac{d}{d t} \int_{\Omega} q_{k}^{2} e^{\rho w} \leq-\int_{\Omega}\left|\nabla q_{k}\right|^{2} e^{\rho w}+C\left(\int_{\Omega} q_{k}^{3}+k \int_{\Omega} q_{k}^{2}+k^{2} \int_{\Omega} q_{k}\right),
$$

where the constant $C=C\left(\lambda, \rho,\left\|w_{0}\right\|_{L^{\infty}(\Omega)}, \beta\right)$. Here, we add the term $\int_{\Omega} q_{k}^{2}$ to both sides to get

$$
\frac{d}{d t} \int_{\Omega} q_{k}^{2} e^{\rho w}+2 \int_{\Omega} q_{k}^{2} \leq-2 \int_{\Omega}\left|\nabla q_{k}\right|^{2}+C \int_{\Omega} q_{k}^{3}+C(k+1) \int_{\Omega} q_{k}^{2}+k^{2} \int_{\Omega} q_{k} .
$$

Then, the Gagliardo-Nirenberg inequality and Young inequality yield

$$
C\left\|q_{k}\right\|_{L^{3}(\Omega)}^{3} \leq C \mid\left\|q_{k}\right\|_{L^{3}(\Omega)}^{2} \leq\left\|q_{k}\right\|_{H^{1}(\Omega)}^{4 / 3}\left\|q_{k}\right\|_{L^{1}(\Omega)}^{2 / 3} \leq \frac{\epsilon}{3}\left\|q_{k}\right\|_{H^{1}(\Omega)}^{2}+C(\epsilon)\left\|q_{k}\right\|_{L^{1}(\Omega)}^{2} .
$$

By using the Gagliardo-Nirenberg inequality again, we have:

$$
\begin{aligned}
C(k+1) \int_{\Omega} q_{k}^{2} & \leq C(k+1)\left\|q_{k}\right\|_{L^{1}(\Omega)}\left\|q_{k}\right\|_{H^{1}(\Omega)} \\
& \leq \frac{\epsilon}{3}\left\|q_{k}\right\|_{H^{1}(\Omega)}^{2}+C(\epsilon)(k+1)^{2}\left\|q_{k}\right\|_{L^{1}(\Omega)} .
\end{aligned}
$$

For the term $\left\|q_{k}\right\|_{L^{1}(\Omega)}$, we apply the Hölder inequality and Sobolev inequality to obtain

$$
\begin{aligned}
C(k+1)^{2}\left\|q_{k}\right\|_{L^{1}(\Omega)} & \leq C(k+1)^{2}\left\|1_{\Omega_{k}}\right\|_{L^{4 / 3}(\Omega)}\left\|q_{k}\right\|_{L^{4}(\Omega)} \\
& \leq \frac{\epsilon}{3}\left\|q_{k}\right\|_{H^{1}(\Omega)}^{2}+C(\epsilon)(k+1)^{4}\left|\Omega_{k}\right|^{3 / 2} .
\end{aligned}
$$


Due to one-dimension setting, we use the embedding $W^{1,2}(\Omega) \hookrightarrow L^{\infty}(\Omega)$ to get same inequality above. Therefore, Equation (24) can be rewritten as:

$$
\frac{d}{d t} \int_{\Omega} q_{k}^{2} e^{\rho w}+(2-\epsilon) \int_{\Omega} q_{k}^{2} \leq(\epsilon-2) \int_{\Omega}\left|\nabla q_{k}\right|^{2}+(k+1)^{4} C(\epsilon)\left|\Omega_{k}\right|^{3 / 2} .
$$

Now, we set

$$
y(t)=\int_{\Omega} q_{k}^{2} e^{\rho w} .
$$

and choose $\epsilon=1$. By following proof of Lemma 3.13 in [20], we get

$$
y^{\prime}+y(t) \leq C\left|\Omega_{k}\right|^{5 / 4},
$$

where $C$ is independent of $k$ and $t$. We solve this inequality to get

$$
y(t) \leq C t e^{-t}\left|\Omega_{k}\right|^{5 / 4}+y(0) .
$$

We choose positive constant $\eta$ which is sufficiently small and $K$ large enough such that when $k_{0}<j<k<K,\left|\Omega_{k}\right|^{5 / 4}>1>\eta$. As a consequence, we have

$$
y(t) \leq\left(C+\frac{y(0)}{\eta}\right)\left(\sup _{t \in\left[0, T_{\max }\right)}\left|\Omega_{k}(t)\right|\right)^{5 / 4} .
$$

From previous lemma, we know

$$
\left\|q_{k}(\cdot, t)\right\|_{L^{2}(\Omega)}^{2} \leq C y(t) \leq C\left(\sup _{t \in\left[0, T_{\max }\right)}\left|\Omega_{k}(t)\right|\right)^{5 / 4} .
$$

On the other hand, $\Omega_{j}(t) \subset \Omega_{k}(t)$ if $j>k>1$. Thus,

$$
\left\|q_{k}(\cdot, t)\right\|_{L^{2}(\Omega)}^{2} \geq \int_{\Omega} q_{k}^{2} \geq(j-k)^{2}\left|\Omega_{j}(t)\right| .
$$

Therefore, we have

$$
(j-k)^{2} \sup _{t \in\left[0, T_{\max }\right)}\left|\Omega_{j}(t)\right| \leq C\left(\sup _{t \in\left[0, T_{\max }\right)}\left|\Omega_{k}(t)\right|\right)^{5 / 4}
$$

Let $\varphi(s)=\sup _{t \in\left[0, T_{\max }\right)}\left|\Omega_{s}(t)\right|$; we rewrite the inequality as

$$
\varphi(j) \leq C(j-k)^{-2}(\varphi(k))^{5 / 4} .
$$

Because $\varphi$ is a non-negative and non-increasing function, Lemma B.1 in Appendix B of [21] implies that there exist $k_{0} \leq \infty$ such that $\varphi\left(k_{0}\right)=0$. Therefore, $q_{k_{0}} \equiv 0$. This completes the proof.

Theorem 2. Let the initial data satisfy Equation (3). Then, there exists a unique globally $L^{\infty}$-bounded positive classic solution to Equation (2) satisfying

$$
(p, w) \in C^{2+\alpha, 1+\frac{\alpha}{2}}(\bar{\Omega} \times[0,+\infty]),
$$

for all $t>0$. 
Proof. By the boundedness of $p$ in $L^{\infty}\left(\left(0, T_{\max }\right), L^{\infty}(\Omega)\right)$ and a bootstrap argument in [20], we can see that Equation (2) indeed admits a globally classic solution from the proof of Lemma 4, which means $T_{\max }=\infty$. Moreover, since $\tau=\min \left\{1, \frac{T_{\max }}{6}\right\}=1$, there exists a constant such that $\|p(\cdot, t)\|_{L^{\infty}(\Omega)}<C$ for all $t>0$.

\subsection{Steady States}

The positive steady states of Equation (2) can be defined by:

$$
\left\{\begin{array}{l}
\Delta p-\rho \operatorname{div}(p \nabla w)+\lambda p(1-p)=0 \\
-\gamma p w^{\beta}=0
\end{array}\right.
$$

with boundary condition

$$
\frac{\partial p}{\partial v}-p \rho \frac{\partial w}{\partial v}=0
$$

By the argument in [20], we have

Lemma 6. ([20]) The positive steady states has solution

$$
(p, w)=(1,0)
$$

or

$$
(p, w)=(0, \tilde{w})
$$

where $\tilde{w}$ is any function in $W^{2, s}(\Omega)$.

\section{3. $L^{\infty}$-Convergence of Solution in Two Dimensions}

In this section, we discuss the asymptotic behavior of solution in the spatially two-dimension of problem in Equations (2) and (3). First, we define the Lyapunov functional

$$
F(p, w):=\frac{\rho}{2 \gamma} \int_{\Omega} w^{-\beta}|\nabla w|^{2}+\int_{\Omega} p(\log p-1) .
$$

Lemma 7. The equality

$$
\frac{d}{d t} F(p, w)=-\int_{\Omega} \frac{|\nabla p|}{p}-\frac{\rho \beta}{2} \int_{\Omega} w^{-1} p|\nabla w|^{2}+\lambda \int_{\Omega} p(1-p) \log p
$$

is valid for all $\beta \geq 1$.

Proof. The simple computation shows that

$$
\frac{d}{d t} \frac{\rho}{2 \gamma} \int_{\Omega} w^{-\beta}|\nabla w|^{2}=-\rho \int_{\Omega} \nabla w \cdot \nabla p-\frac{\rho \beta}{2} \int_{\Omega} w^{-1} p|\nabla w|^{2}
$$

and

$$
\frac{d}{d t} \int_{\Omega} p(\log p-1)=-\int_{\Omega} \frac{|\nabla p|^{2}}{p}+\rho \int_{\Omega} \nabla w \cdot \nabla p+\lambda \int_{\Omega} p(1-p) \log p .
$$

Combining above two equalities, we complete the proof.

Then, we define

$$
G(p, w)=-\int_{\Omega} \frac{|\nabla p|}{p}-\frac{\rho \beta}{2} \int_{\Omega} w^{-1} p|\nabla w|^{2}+\lambda \int_{\Omega} p(1-p) \log p .
$$




\subsection{Asymptotic Behavior When $\beta>1$}

Lemma 8. There exists constant $C>0$ such that

$$
G(p, w) \leq-\int_{\Omega} \frac{|\nabla p|^{2}}{p}-C \int_{\Omega} p|\nabla w|^{2} .
$$

Proof. By solving the $w$-equation, we obtain

$$
w(x, t)=w_{0} e^{-\gamma \int_{0}^{t} p(s) w^{\beta-1} d s}
$$

and thus $w^{-1}>\left\|w_{0}\right\|_{L^{\infty}(\Omega)}^{-1}$ by the positivity of $p$. Now, combining the above inequalities with the fact that $s(1-s) \log s<0$ for all $s>0$, we arrive at Equation (34).

Lemma 9. There exists constant $C>0$ such that

$$
\sup _{t \geq 0} \int_{\Omega}|\nabla w(t)|+\int_{0}^{+\infty} \int_{\Omega} \frac{|\nabla p|^{2}}{p}+\int_{0}^{+\infty} \int_{\Omega} p|\nabla w|^{2} \leq C
$$

Proof. Lemmas 7 and 8 show that

$$
\frac{d}{d t} F(p, w)+\int_{\Omega} \frac{|\nabla p|^{2}}{p}+C \int_{\Omega} p|\nabla w|^{2} \leq 0 .
$$

Then, integrating over time variable, we have

$$
F(p(t), w(t))+\int_{0}^{t} \int_{\Omega} \frac{|\nabla p|^{2}}{p}+\int_{0}^{t} \int_{\Omega} p|\nabla w|^{2} \leq F\left(p_{0}, w_{0}\right) .
$$

Since $\int_{\Omega} p(\log p-1) \geq \int_{0<p<e} p(\log p-1) \geq-|\Omega|\left(e^{-1}+e\right)$, we conclude that

$$
\left\|w_{0}\right\|_{L^{\infty}(\Omega)}^{-\beta} \int_{\Omega}|\nabla w|^{2}+\int_{0}^{t} \int_{\Omega} \frac{|\nabla p|^{2}}{p}+\int_{0}^{t} \int_{\Omega} p|\nabla w|^{2} \leq C,
$$

which implies

$$
\sup _{t \geq 0} \int_{\Omega}|\nabla w(t)|+\int_{0}^{+\infty} \int_{\Omega} \frac{|\nabla p|^{2}}{p}+\int_{0}^{+\infty} \int_{\Omega} p|\nabla w|^{2} \leq C
$$

Lemma 10. If the initial data satisfy Equation (3), then, for any $r \geq 2$,

$$
\lim _{t \rightarrow \infty}\|p(\cdot, t)-\bar{p}\|_{L^{r}(\Omega)}=0 .
$$

Proof. We define $k(t)=\int_{\Omega}|p(\cdot, t)-\bar{p}|^{2}$ and

$$
h(t)=\int_{\Omega}|\nabla p|^{2}+\int_{\Omega} p|\nabla w|^{2}+\int_{\Omega} p(p-1)^{2}+\int_{\Omega}(p-\bar{p})^{2} .
$$

By the proof in [20], it is easy to check that $k(t) \rightarrow 0$, which implies $\lim _{t \rightarrow \infty}\|p(\cdot, t)-\bar{p}(t)\|_{L^{2}(\Omega)}=0$. The Gagliardo-Nirenberg inequality indicates that

$$
\|p(\cdot, t)-\bar{p}(t)\|_{L^{r}(\Omega)} \leq C\|p(\cdot, t)\|_{W^{1,2}(\Omega)}^{1-\frac{2}{r}}\|p(\cdot, t)-\bar{p}(t)\|_{L^{2}(\Omega)}^{\frac{2}{r}} .
$$

We can get Equation (41) immediately with Lemmas 4 and 9. 
Lemma 11. If the initial data satisfy Equation (3), then, for any $r \geq 2$, we have

$$
\lim _{t \rightarrow \infty}\|w(\cdot, t)\|_{L^{r}(\Omega)}=0
$$

and

$$
\lim _{t \rightarrow \infty}|\bar{p}(t)-1|=0
$$

Proof. The proof of Equation (44) is the same as the one in [20] for Lemmas 5.10 and 5.11. We only need to prove Equation (43). With the Poincaré-Wirtinger inequality, we can choose $k \in \mathbb{N}$ such that

$$
\begin{aligned}
\int_{k}^{k+1}\|p(\cdot, s)-\bar{p}(s)\|_{L^{2}(\Omega)}^{2} d s & \leq C \int_{k}^{k+1}\|\nabla p(\cdot, s)\|_{L^{2}(\Omega)}^{2} d s \\
& \leq C \sup _{t \geq 0}\|p(\cdot, t)\|_{L^{\infty}(\Omega)} \int_{k}^{k+1} \int_{\Omega} \frac{|\nabla p(\cdot, s)|^{2}}{p(s)} d s,
\end{aligned}
$$

Then, Lemma 10 implies that

$$
\int_{\Omega} \int_{k}^{k+1}|p(\cdot, s)-\bar{p}(s)|^{2} d s=\int_{k}^{k+1}|| p(s)-\bar{p}(s) \|_{L^{2}(\Omega)}^{2} \rightarrow 0
$$

as $t \rightarrow \infty$. Now, we define $p_{k}=\int_{k}^{k+1}\|p(s)-\bar{p}(s)\|_{L^{2}(\Omega)}^{2}$. Then, Equation (45) indicates that $p_{k} \rightarrow 0$ in $L^{1}(\Omega)$ as $k \rightarrow \infty$. Now, we choose subsequence $\left(k_{i}\right)$ with $i \in \mathbb{N}$ such that $p_{k_{i}} \rightarrow 0$ for every $x \in \Omega / Q$ with $Q \subset \Omega,|Q|=0$ as $i \rightarrow \infty$ and a constant $k_{i_{0}}$ such that for all $i \geq i_{0}$

$$
p_{k_{i}}<\frac{1}{4 \gamma(\beta-1) n^{2}(t)}
$$

where $n(t)=\max \left\{k_{i}+1,[t]\right\}$. Because of Equation (44), we can pick some $k_{i_{1}}>0$ such that for all $i \geq i_{1}$

$$
\int_{k_{i}}^{k_{i}+1} \bar{p}(s) d s>1 / 2
$$

Then, for any $x \in \Omega / Q$

$$
\begin{aligned}
|w(x, t)| & =\left(\frac{1}{\gamma(\beta-1) \sum_{i=0}^{n(t)} \int_{k_{i}}^{k_{i+1}} p(s) d s+w_{0}^{1-\beta}}\right)^{\frac{1}{\beta-1}} \\
& <\left(\frac{1}{-\gamma(\beta-1) \sum_{i=M}^{n(t)}\left(\int_{k_{i}}^{k_{i+1}}|p(x, s)-\bar{p}(s)| d s+\sum_{k=N}^{n(t)} \int_{k_{i}}^{k_{i+1}} \bar{p}(s) d s\right)}\right)^{\frac{1}{\beta-1}} \\
& <\left(\frac{1}{-\gamma(\beta-1) \sum_{i=M}^{n(t)}\left(\left(\int_{k_{i}}^{k_{i+1}}|p(x, s)-\bar{p}(s)|^{2} d s\right)^{1 / 2}+\sum_{i=M}^{n(t)} \int_{k_{i}}^{k_{i+1}} \bar{p}(s) d s\right)}\right)^{\frac{1}{\beta-1}},
\end{aligned}
$$

where $M=\max \left\{k_{i_{0}}, k_{i_{1}}\right\}$. Now, we can conclude that

$$
w(x, t) \rightarrow 0 \quad \text { in } \Omega .
$$

as $t \rightarrow \infty$. On the other hand, $|w(x, t)| \leq\left\|w_{0}\right\|_{L^{\infty}(\Omega)}$ implies that $\lim _{t \rightarrow \infty}\|w(\cdot, t)\|_{L^{r}(\Omega)}=0$ by the dominated convergence theorem.

Then, we use the well-known $L^{p}-L^{q}$ estimates of heat semigroup and the Gagliardo-Nirenberg inequality to show the $L^{\infty}$-convergence of solution. 
Lemma 12. There exists a constant $C$ such that

$$
\|\nabla p(\cdot, t)\|_{L^{4}(\Omega)} \leq C
$$

Proof. We notice that $q=p e^{-\rho w}$ satisfies

$$
\frac{d}{d t} q=\Delta q+\nabla w \cdot \nabla q+\lambda q\left(1-e^{\rho w} q\right)+\rho \gamma q^{2} e^{\rho w} w^{\beta} .
$$

Now, we define $h(q, w)=\lambda q\left(1-e^{\rho w} q\right)+\rho \gamma q^{2} e^{\rho w} w^{\beta}$. Applying variation-of-constant formula to $q$-equation yields

$$
q=e^{t \Delta} q(\cdot, 0)+\int_{0}^{t} e^{(t-s) \Delta}(\nabla w \cdot \nabla q+h(q, w)) .
$$

Then, the well-known $L^{p}-L^{q}$ estimate of heat semigroup shows that

$$
\begin{aligned}
\|\nabla q(\cdot, t)\|_{L^{4}(\Omega)} \leq C\left(e^{-\lambda_{1} t}\|\nabla q(\cdot, 0)\|_{L^{4}(\Omega)}\right. & +\int_{0}^{t}\left(1+(t-s)^{-\frac{3}{4}}\right) e^{-\lambda_{1}(t-s)}\|\nabla w \cdot \nabla q\|_{L^{2}(\Omega)} d s \\
& +\int_{0}^{t}\left(1+(t-s)^{-\frac{3}{4}}\right) e^{-\lambda_{1}(t-s)}\|h(q, w)\|_{L^{2}(\Omega)} d s
\end{aligned}
$$

Then, the Hölder inequality with the boundedness of $w$ and $p$ show that

$$
\|\nabla q(\cdot, t)\|_{L^{4}(\Omega)} \leq C_{1}+C_{2} \int_{0}^{t}\left(1+(t-s)^{-\frac{3}{4}}\right) e^{-\lambda_{1}(t-s)}\|\nabla q\|_{L^{4}(\Omega)} d s
$$

where $C_{1}$ and $C_{2}$ are independent of $t$. Then, Gronwall inequality yields

$$
\|\nabla q(\cdot, t)\|_{L^{4}(\Omega)} \leq C e^{\int_{0}^{t}\left(1+(t-s)^{-\frac{3}{4}}\right) e^{-\lambda_{1}(t-s)} d s} \leq C e^{\frac{1}{\lambda_{1}}+\Gamma\left(\frac{1}{4}\right)},
$$

where $\Gamma$ represents the gamma function.

Lemma 13. If the initial data satisfy Equation (3), then we have

$$
\lim _{t \rightarrow \infty}\|p(\cdot, t)-1\|_{L^{\infty}(\Omega)}=0
$$

Proof. The $L^{2}$-convergence of $p$ can be proved by the argument in Lemmas 5.9, 5.10, and 5.11 in [20]. Furthermore, the Gagliardo-Nirenberg inequality implies that

$$
\|p(\cdot, t)-1\|_{L^{\infty}(\Omega)} \leq C\|p(\cdot, t)\|_{W^{1,4}(\Omega)}^{1 / 2}\|p(\cdot, t)-1\|_{L^{2}(\Omega)}^{1 / 2} .
$$

Then, Equation (53) follows by the boundedness of $p$ and Lemma 12.

Now, we establish the explicit decay rate of $p(x, t)-1$ and $w(x, t)$ with respect to $L^{\infty}$-norm.

Lemma 14. For any $\epsilon \in(0,1)$, there exist $t_{\epsilon}$ and constant $C(\epsilon)$ such that for all $t>t_{\epsilon}$

$$
\int_{t_{\epsilon}}^{t}\left(\gamma(\beta-1)\left(s-t_{\epsilon}\right)+\left\|w_{0}\right\|_{L^{\infty}(\Omega)}^{1-\beta}\right)^{\frac{2}{\beta-1}} \int_{\Omega}|\nabla w|^{2} d s \leq C(\epsilon) .
$$

Proof. The explicit expression of $w(x, t)$ shows that

$$
|\nabla w|^{2} \leq 2\left(\int_{0}^{t}(\beta-1) \gamma p(\cdot, s) d s+\left\|w_{0}\right\|_{L^{\infty}(\Omega)}^{1-\beta}\right)^{\frac{2 \beta}{1-\beta}}\left(\gamma^{2} \int_{0}^{t}|\nabla p(\cdot, s)|^{2} d s+\sup _{x \in \bar{\Omega}}\left\{w_{0}^{-2 \beta}\left|\nabla w_{0}\right|^{2}\right\}\right) .
$$


Then, we can choose positive $\epsilon \in(0,1)$ and $t_{\epsilon}$ such that

$$
p(x, t)>1-\epsilon
$$

for all $x \in \Omega$ whenever $t>t_{\epsilon}$. Now, we multiply the term $\left(\gamma(\beta-1)(1-\epsilon)\left(t-t_{\epsilon}\right)+\left\|w_{0}\right\|_{L^{\infty}(\Omega)}^{1-\beta}\right)$ on both side and integrate over the interval $\left[t_{\epsilon}, t\right]$ to obtain

$$
\begin{aligned}
& \int_{t_{\epsilon}}^{t}\left(\gamma(\beta-1)(1-\epsilon)\left(s-t_{\epsilon}\right)+\left\|w_{0}\right\|_{L^{\infty}(\Omega)}^{1-\beta}\right)^{\frac{2 \beta}{\beta-1}} \int_{\Omega}|\nabla w|^{2} d s \\
\leq & \frac{2 C}{\gamma^{2}(1-\epsilon)^{2}(\beta-1)^{2}} \int_{t_{\epsilon}}^{t}\left(s-t_{\epsilon}+\frac{\left\|w_{0}\right\|_{L^{\infty}(\Omega)}^{1-\beta}}{\gamma(\beta-1)(1-\epsilon)}\right)^{-2} d s,
\end{aligned}
$$

which leads to Equation (54).

Lemma 15. For any $\epsilon \in(0,1)$, there exist constant $C(\epsilon)>0$ and $t_{\epsilon}>0$ such that

$$
\|p(\cdot, t)-1\|_{L^{2}(\Omega)} \leq C(\epsilon)\left(\left\|w_{0}\right\|_{L^{\infty}(\Omega)}^{1-\beta}+\gamma(\beta-1)(1-\epsilon)\left(t-t_{\epsilon}\right)\right)^{\frac{1}{1-\beta}}
$$

for all $t>t_{\epsilon}$.

Proof. We choose $\epsilon \in(0,1)$ and $t_{\epsilon}$ such that $p(x, t)>1-\epsilon$ for all $x \in \mathbb{R}^{n}$. By multiplying the $p$-equation with $p-1$ and integrating over $\Omega$, we have

$$
\begin{aligned}
\frac{1}{2} \frac{d}{d t} \int_{\Omega}(p-1)^{2} & \leq-\frac{1}{2} \int_{\Omega}|\nabla p|^{2}+C \rho \int_{\Omega}|\nabla w|^{2}-\lambda(1-\epsilon) \int_{\Omega}(p-1)^{2} \\
& \leq C \rho \int_{\Omega}|\nabla w|^{2}-\lambda(1-\epsilon) \int_{\Omega}(p-1)^{2} .
\end{aligned}
$$

Now, we choose $t_{2}=\frac{1}{2 \lambda(\beta-1)(1-\epsilon)}\left(\max \left\{\frac{1}{2}-\left\|w_{0}\right\|_{L^{\infty}(\Omega)^{\prime}}^{1-\beta} 0\right\}\right)+t_{\epsilon}$. Then, for any $t>t_{2}$

$$
\begin{gathered}
\int_{t_{2}}^{t} \frac{d}{d s}\left(\left\|w_{0}\right\|_{L^{\infty}(\Omega)}^{1-\beta}+\gamma(\beta-1)(1-\epsilon)\left(s-t_{\epsilon}\right)\right)^{\frac{2}{\beta-1}} \int_{\Omega}(p-1)^{2} d s \\
\leq \int_{t_{\epsilon}}^{t}\left(\left\|w_{0}\right\|_{L^{\infty}(\Omega)}^{1-\beta}+\gamma(\beta-1)(1-\epsilon)\left(s-t_{\epsilon}\right)\right)^{\frac{2}{\beta-1}}\left(\frac{d}{d t} \int_{\Omega}(p-1)^{2}+2 \lambda(1-\epsilon) \int_{\Omega}(p-1)^{2}\right) d s .
\end{gathered}
$$

This implies that

$$
\begin{array}{r}
\left(\left\|w_{0}\right\|_{L^{\infty}(\Omega)}^{1-\beta}+\gamma(\beta-1)(1-\epsilon)\left(t-t_{\epsilon}\right)\right)^{\frac{2}{\beta-1}} \int_{\Omega}(p-1)^{2} \\
\leq 2 C \rho \int_{t_{\epsilon}}^{t}\left(\left\|w_{0}\right\|_{L^{\infty}(\Omega)}^{1-\beta}+\gamma(\beta-1)(1-\epsilon)(s-t)\right)^{\frac{2}{\beta-1}} \int_{\Omega}|\nabla w|^{2} \\
+\left(\left\|w_{0}\right\|_{L^{\infty}(\Omega)}^{1-\beta}+\gamma(\beta-1)(1-\epsilon)\left(t_{2}-t_{\epsilon}\right)\right)^{\frac{2}{\beta-1}} \int_{\Omega}\left(p\left(\cdot, t_{2}\right)-1\right)^{2},
\end{array}
$$

which leads to Equation (56).

Lemma 16. For any $\epsilon \in(0,1)$, there exist constant $C(\epsilon)$ and $t_{\epsilon}$ such that for all $t>t_{\epsilon}$

$$
\|p(\cdot, t)-1\|_{L^{\infty}(\Omega)} \leq C(\epsilon)\left(\left\|w_{0}\right\|_{L^{\infty}(\Omega)}^{1-\beta}+\gamma(\beta-1)(1-\epsilon)\left(t-t_{\epsilon}\right)\right)^{\frac{1}{2(1-\beta)}}
$$

for all $t>t_{\epsilon}$. 
Proof. By the Gagliardo-Nirenberg inequality in the two-dimensional setting, we can obtain

$$
\|p(\cdot, t)-1\|_{L^{\infty}(\Omega)} \leq C\|p(\cdot, t)\|_{W^{1,4}}^{1 / 2}\|p(\cdot, t)-1\|_{L^{2}(\Omega)}^{1 / 2} .
$$

The Gagliardo-Nirenberg inequality still has a similar form in the one-dimensional setting. Then, Lemmas 5, 12, and 15 imply Equation (57).

Lemma 17. For any $\epsilon \in(0,1)$, there exist constant $C>0$ and $t_{\epsilon}$ such that

$$
\|w(\cdot, t)\|_{W^{1,4}(\Omega)} \leq C\left(\gamma(\beta-1)(1-\epsilon)\left(t-t_{\epsilon}\right)+\left\|w_{0}\right\|_{L^{\infty}(\Omega)}^{1-\beta}\right)^{\frac{1}{1-\beta}} .
$$

for all $t>t_{\epsilon}$.

Proof. Now, we choose the same $\epsilon$ in Lemma 14. Then, the explicit expression of $w$ implies that

$$
\int_{\Omega}|\nabla w|^{4} \leq C\left(\left\|w_{0}\right\|_{L^{\infty}(\Omega)}^{1-\beta}\right)^{-4}\left(\gamma(\beta-1)\left(t-t_{\epsilon}\right)+\left\|w_{0}\right\|_{L^{\infty}(\Omega)}^{1-\beta}\right)^{\frac{4}{1-\beta}}
$$

Then, we obtain Equation (58).

Collecting all the lemmas above, we infer that

Theorem 3. If the initial data satisfy Equation (3), then the solution satisfies

$$
\lim _{t \rightarrow \infty}\|p(\cdot, t)-1\|_{L^{\infty}(\Omega)}+\|w(\cdot, t)\|_{L^{\infty}(\Omega)}=0 .
$$

Moreover, for some $\epsilon \in(0,1)$, there exists $t_{\epsilon}>0$ such that for any $t>t_{\epsilon}$

$$
\|p(\cdot, t)-1\|_{L^{\infty}(\Omega)} \leq C(\epsilon)\left(\left\|w_{0}\right\|_{L^{\infty}(\Omega)}^{1-\beta}+\gamma(\beta-1)\left(t-t_{\epsilon}\right)\right)^{\frac{1}{2(1-\beta)}} .
$$

and

$$
\|w(\cdot, t)\|_{W^{1,4}} \leq C(\epsilon)\left(\left\|w_{0}\right\|_{L^{\infty}(\Omega)}^{1-\beta}+\gamma(\beta-1)\left(t-t_{\epsilon}\right)\right)^{\frac{1}{1-\beta}}
$$

3.2. Asymptotic Behavior When $\beta=1$

By checking the proof of Lemmas 10 and 11, a similar result is also valid for $\beta=1$ which can be stated as follows

Theorem 4. If $\beta=1$, we have

$$
\lim _{t \rightarrow \infty}|| p(\cdot, t)-1 \|_{L^{\infty}(\Omega)}=0
$$

and

$$
\lim _{t \rightarrow \infty}\|w(\cdot, t)\|_{L^{\infty}(\Omega)}=0 .
$$

At this position, we focus on the explicit decay rate of solutions.

Lemma 18. For any $\epsilon \in(0,1)$, there exists $C>0$ such that

$$
\int_{\Omega}|\nabla w|^{2} \leq C e^{-2 \gamma(1-\epsilon) t} .
$$


Proof. It is observed that

$$
|\nabla w|^{2} \leq 4\left|\nabla w_{0}\right|^{2} e^{-2 \gamma \int_{0}^{t} p(s) d s}+4 \gamma^{2}\left|w_{0}\right|^{2} e^{-2 \gamma \int_{0}^{t} p(s) d s} \int_{0}^{t}|\nabla p(s)|^{2} .
$$

Now, Equation (63) implies that there exist $\epsilon>0$ and $t_{\epsilon}$ such that $p(x, t)>1-\epsilon$ whenever $t>t_{\epsilon}$ for all $x \in \Omega$. By integrating Equation (66) over $\Omega$, we get

$$
\int_{\Omega}|\nabla w|^{2} \leq C e^{-2 \gamma(1-\epsilon) t}\left(\int_{\Omega}\left|\nabla w_{0}\right|^{2}+\left\|w_{0}\right\|_{L^{\infty}(\Omega)}^{2} \sup _{t \geq 0}\|p(t)\|_{L^{\infty}(\Omega)} \int_{0}^{\infty} \int_{\Omega} \frac{|\nabla p|^{2}}{p}\right)
$$

which implies Equation (65).

Lemma 19. For any $\epsilon \in(0, \min \{1, \gamma, \lambda\})$, there exists $C(\epsilon)>0$ such that

$$
\|p(\cdot, t)-1\|_{L^{2}(\Omega)} \leq C(\epsilon) e^{-(\min \{1, \gamma, \lambda\}-\epsilon) t} .
$$

Proof. We pick $\epsilon>0$ such that $p(x, t)>1-\epsilon$ for all $x \in \Omega$ with $t>t_{\epsilon}$. Hence, we multiply the $p$-equation by $(p-1)$ and integrate the result over $\Omega$ to obtain

$$
\begin{array}{r}
\frac{1}{2} \frac{d}{d t} \int_{\Omega}(p-1)^{2}=-\int_{\Omega}|\nabla p|^{2}+\rho \int_{\Omega} p \nabla w \cdot \nabla p-\lambda \int_{\Omega} p(p-1)^{2} \\
\leq-\frac{1}{2} \int|\nabla p|^{2}+c_{4} \int_{\Omega}|\nabla w|^{2}-\lambda(1-\epsilon) \int_{\Omega}(p-1)^{2}
\end{array}
$$

for all $t>t_{\epsilon}$. The Gronwall inequality shows that

$$
\begin{aligned}
\int_{\Omega}(p(t)-1)^{2} & \leq e^{-2 \lambda(1-\epsilon)\left(t-t_{\epsilon}\right)} \int_{\Omega}\left(p\left(t_{\epsilon}\right)-1\right)^{2}+c_{4} \int_{0}^{t} e^{-2 \lambda(1-\epsilon)(t-s)} \int_{\Omega}|\nabla w|^{2} \\
& \leq e^{2 \lambda(1-\epsilon) t_{\epsilon}} \int_{\Omega}\left(p\left(t_{\epsilon}\right)-1\right)^{2} e^{-2 \lambda(1-\epsilon) t}+c_{4} C t e^{-2 \min \{1, \gamma, \lambda\}(1-\epsilon) t} \\
& \leq c_{5}(\epsilon)(1+t) e^{-2 \min \{1, \gamma, \lambda\}(1-\epsilon) t} .
\end{aligned}
$$

Then, we have

$$
\begin{aligned}
\int_{\Omega}(p(t)-1)^{2} & \leq c_{5}(\epsilon)(1+t) e^{2(\min \{1, \gamma, \lambda\}-1) \epsilon t} e^{-2(\min \{1, \gamma, \lambda\}-\epsilon) t} \\
& \leq C(\epsilon) e^{-2(\min \{1, \gamma, \lambda\}-\epsilon) t}
\end{aligned}
$$

which completes the proof.

By checking the proof of Lemmas 13, 15 and 16, we can establish the following explicit decay rate.

Theorem 5. If the initial data satisfy Equation (3), for any $\epsilon \in(0, \min \{1, \gamma, \lambda\})$, we have

$$
\|p(\cdot, t)-1\|_{L^{\infty}(\Omega)} \leq C e^{-\left(\frac{1}{2} \min \{1, \gamma, \lambda\}-\epsilon\right) t}
$$

and

$$
\|w(\cdot, t)\|_{L^{\infty}(\Omega)} \leq C e^{-2 \gamma(1-\epsilon) t}
$$

for any $\epsilon \in\left(0, \frac{1}{2} \min \{1, \gamma, \lambda\}\right)$. 


\section{4. $L^{\infty}$-Convergence in One Dimensions}

In this section, we establish the explicit decay rate of $p(x, t)-1$ and $w(x, t)$ for $\beta=1$ and $\beta>1$ in one-dimensional setting respectively. In should be mentioned here that the results in previous subsection are still valid in the one-dimensional case.

The following lemma plays a crucial role in establishing the uniformly convergence of $p(x, t)-1$ when $\beta \geq 1$.

Lemma 20. There exists a constant $C>0$ such that:

$$
\int_{0}^{\infty} \int_{\Omega} e^{\rho w}|\nabla q|^{2} \leq C
$$

where $q=p e^{-\rho w}$.

Proof. By using the Cauchy inequality, we have

$$
e^{\rho w}|\nabla q|^{2} \leq 2 e^{-\rho w}|\nabla p|^{2}+2 p^{2} e^{-\rho w} \rho^{2}|\nabla w|^{2} .
$$

Then, we integrate it over $\Omega \times(0, \infty)$ to obtain

$$
\begin{aligned}
\int_{0}^{\infty} \int_{\Omega} e^{\rho w}|\nabla q|^{2} & \leq 2 \int_{0}^{\infty} \int_{\Omega}|\nabla p|^{2}+2 \rho^{2} \sup _{t \geq 0}|| p(t) \|_{L^{\infty}(\Omega)} \int_{0}^{\infty} \int_{\Omega} p|\nabla w|^{2} \\
& \leq 2\left(1+\rho^{2}\right) \sup _{t \geq 0}\|p(t)\|_{L^{\infty}(\Omega)}\left(\int_{0}^{+\infty} \int_{\Omega} \frac{|\nabla p|^{2}}{p}+\int_{0}^{\infty} \int_{\Omega} p|\nabla w|^{2}\right),
\end{aligned}
$$

which implies Equation (71).

Lemma 21. There exists a constant $C$ such that

$$
\frac{d}{d t} \int_{\Omega} e^{\rho w}|\nabla q|^{2}+\frac{1}{2} \int_{\Omega} e^{\rho w} q_{t}^{2} \leq C\left(\int_{\Omega} e^{\rho w}|\nabla q|^{2}+\int_{\Omega} p(p-1)^{2}+\int_{\Omega} p w^{\beta}\right)
$$

for all $\beta \geq 1$.

Proof. By differentiating $q$-equation with respect to time variable, we can easily obtain

$$
\int_{\Omega} e^{\rho w} q_{t}^{2}+\int_{\Omega} e^{\rho w} \nabla q \cdot \nabla q_{t}=\lambda \int_{\Omega} q\left(1-q e^{\rho w}\right) q_{t} e^{\rho w}+\int_{\Omega} e^{2 \rho w} \gamma \rho w w^{\beta} q^{2} .
$$

By the fact that

$$
\int_{\Omega} e^{\rho w} \nabla q \cdot \nabla q_{t}=\frac{1}{2} \frac{d}{d t} \int_{\Omega} e^{\rho w}|\nabla q|^{2}+\frac{\gamma \rho}{2} \int_{\Omega} e^{2 \rho w} q w w^{\beta}|\nabla q|^{2},
$$

we have

$$
\int_{\Omega} e^{\rho w} \nabla q \cdot \nabla q_{t} \geq \frac{1}{2} \frac{d}{d t} \int_{\Omega} e^{\rho w}|\nabla q|^{2}
$$

We notice that

$$
\begin{aligned}
& \int_{\Omega} e^{\rho w} q_{t}\left(\left(\lambda q\left(1-q e^{\rho w}\right)+\gamma \rho e^{\rho w} q^{2} w^{\beta}\right)\right. \\
= & \lambda \int_{\Omega} p q_{t}(1-p)+\rho \gamma \int_{\Omega} q_{t} p^{2} w^{\beta} \\
\leq & \epsilon \int_{\Omega} e^{\rho w} q_{t}^{2}+C(\epsilon)\left(\sup _{t \geq 0}\|p(t)\|_{L^{\infty}(\Omega)} \int_{\Omega} p(1-p)^{2}+\sup _{t \geq 0}\|p(t)\|_{L^{\infty}(\Omega)}^{3}\left\|w_{0}\right\|_{L^{\infty}(\Omega)} \int_{\Omega} p w^{\beta}\right) .
\end{aligned}
$$


Thus, the inequality in Equation (72) can be deduced by Equations (73) and (74) and Lemma 5 by choosing $\epsilon=\frac{1}{2}$.

Lemma 22. If the initial data satisfy Equation (3), then we have

$$
\int_{0}^{\infty} \int_{\Omega} p w^{\beta} \leq \frac{\left\|w_{0}\right\|_{L^{1}(\Omega)}}{\gamma}
$$

Proof. By integrating the $w$-equation in time variable, we have

$$
\gamma \int_{0}^{t} \int_{\Omega} p w^{\beta}=\int_{\Omega} w_{0}-\int_{\Omega} w \leq\left\|w_{0}\right\|_{L^{1}(\Omega)}
$$

for arbitrary $t>0$, which leads to Equation (75).

\subsection{Asymptotic Behavior When $\beta>1$}

Now, we focus our attention on the decay property of solutions for $\beta>1$.

Lemma 23. If the initial data satisfy Equation (3), then we have:

$$
\lim _{t \rightarrow+\infty}\|w(\cdot, t)\|_{L^{\infty}(\Omega)}=0
$$

Proof. By the Poincaré-Wirtinger inequality, we have

$$
\begin{aligned}
\int_{0}^{t}\|p(s)-\bar{p}(s)\|_{L^{\infty}(\Omega)}^{2} & \leq \int_{0}^{t}\|p(s)-\bar{p}(s)\|_{W^{1,2}(\Omega)} d s \\
& \leq C \int_{0}^{t}\|\nabla p\|_{L^{2}(\Omega)}^{2} d s \leq C \sup _{t \geq 0}\|p\|_{L^{\infty}(\Omega)} \int_{0}^{\infty} \int_{\Omega} \frac{|\nabla p(\cdot, s)|^{2}}{p} d s .
\end{aligned}
$$

This indicates that the term $\int_{0}^{t}\|p(\cdot, s)-\bar{p}(s)\|_{L^{\infty}(\Omega)}^{2}$ is bounded. Now, we choose $t_{0}$ such that overline $p(t)>\frac{1}{2}$ for all $t>t_{0}$ We notice

$$
\begin{array}{r}
w_{0}^{1-\beta}+(\beta-1) \gamma \int_{0}^{t} p(s) d s \geq(\beta-1) \gamma\left(\frac{1}{2}-\eta\right) t \\
+|| w_{0} \|_{L^{\infty}(\Omega)}^{1-\beta}-\frac{(\beta-1) \gamma}{\eta} \int_{0}^{t}|p(s)-\bar{p}(s)|^{2} d s-\frac{(\beta-1) \gamma}{2} t_{0} .
\end{array}
$$

Then, for any $t>\max \left\{\frac{(\beta-1) \gamma t_{0}+\frac{2(\beta-1) \gamma C}{\eta}-2\left\|w_{0}\right\|_{L^{\infty}(\Omega)}^{1-\beta}}{(\beta-1) \gamma(1-2 \eta)}, 0\right\}=t_{1}$, we pick $\eta<\frac{1}{2}$ to get

$$
\|w(\cdot, t)\|_{L^{\infty}(\Omega)} \leq\left(\gamma(\beta-1)\left(\frac{1}{2}-\eta\right) t-\frac{1}{2}(\beta-1) t_{0}-\frac{C(\beta-1) \gamma}{\eta}+\left\|w_{0}\right\|_{L^{\infty}(\Omega)}^{1-\beta}\right)^{\frac{1}{1-\beta}} .
$$

Then, the assertion now follows from Equation (78).

Lemma 24. If the initial data satisfy Equation (3), then we have

$$
\lim _{t \rightarrow+\infty}\|p(\cdot, t)-1\|_{L^{\infty}(\Omega)}=0 .
$$

Proof. We define

$$
k(t):=\int_{\Omega} e^{\rho w}|\nabla q|^{2}
$$


and $h(t):=\int_{\Omega} e^{\rho w}|\nabla q|^{2}+\int_{\Omega} p|\nabla w|^{2}+\int_{\Omega} p w^{\beta}+\int_{\Omega} p(p-1)^{2} \in L^{1}(0, \infty)$. Then, Lemma 21 implies $k(t) \rightarrow 0$ as $t \rightarrow \infty$.

Now, the Poincaré-Wirtinger inequality and the Sobolev embedding theorem show that

$$
\|q(t)-\bar{q}(t)\|_{L^{\infty}(\Omega)} \leq C\|\nabla q(t)\|_{L^{2}(\Omega)} .
$$

Then, Lemma 20 implies that

$$
\lim _{t \rightarrow \infty}\|q(t)-\bar{q}(t)\|_{L^{\infty}(\Omega)}=0
$$

and Lemmas 10 and 11 show

$$
p e^{\rho w}\left(\cdot, t_{j}\right) \rightarrow e^{\rho} \text { a.e. as } j \rightarrow \infty
$$

for sequence $\left\{t_{j}\right\}$ with $j \in \mathbb{N}$. Meanwhile, the $L^{\infty}$-boundedness of $p$ implies that

$$
\left|q\left(\cdot, t_{j}\right)\right|<\sup _{j \geq 0}\left\|q\left(\cdot, t_{j}\right)\right\|_{L^{\infty}(\Omega)} \leq C .
$$

Then, the dominated convergence theorem and inequality below

$$
\left|\bar{q}(t)-e^{-\rho}\right| \leq \frac{1}{|\Omega|} \int_{\Omega}\left|q(x, t)-e^{-\rho}\right|
$$

yield

$$
\lim _{t \rightarrow \infty}\left|\bar{q}(t)-e^{-\rho}\right|=0
$$

Then, we have

$$
\begin{aligned}
\|p(t)-1\| \|_{L^{\infty}(\Omega)} & \leq\left\|e^{-\rho}\left(e^{\rho w+2 \rho}(q-\bar{q})+e^{\rho w+2 \rho}\left(\bar{q}-e^{-\rho}\right)+e^{\rho w+1}-e^{\rho}\right)\right\|_{L^{\infty}(\Omega)} \\
& \leq e^{\rho\left(2+\left\|w_{0}\right\|_{L^{\infty}(\Omega)}\right)}\left(\|q(t)-\bar{q}(t)\|_{L^{\infty}(\Omega)}+\left|\bar{q}(t)-e^{-\rho}\right|\right)+\left.\left\|e^{\rho w}-1\right\|\right|_{L^{\infty}(\Omega)} \\
& \leq e^{\rho\left(2+\left\|w_{0}\right\|_{L^{\infty}(\Omega)}\right)}\left(\|q(t)-\bar{q}(t)\|_{L^{\infty}(\Omega)}+\left|\bar{q}(t)-e^{-\rho}\right|+C\|w\|_{L^{\infty}(\Omega)}\right) .
\end{aligned}
$$

Thus, Lemma 24 and Equations (82) and (85) yield Equation (79).

The explicit polynomial decay rate can be established by the argument in two-dimensional setting which can be stated as

Lemma 25. If the initial data satisfy Equation (3), then, for any $\epsilon \in(0,1)$, there exist $t_{\epsilon}>0$ such that for all $t>t_{\epsilon}>0$

$$
\|p(\cdot, t)-1\|_{L^{\infty}(\Omega)} \leq C(\epsilon)\left(\left\|w_{0}\right\|_{L^{\infty}(\Omega)}^{1-\beta}+\gamma(\beta-1)(1-\epsilon)\left(t-t_{\epsilon}\right)\right)^{\frac{1}{2(1-\beta)}}
$$

and

$$
\|w(\cdot, t)\|_{W^{1,2}(\Omega)} \leq C\left(\gamma(\beta-1)(1-\epsilon)\left(t-t_{\epsilon}\right)+\left\|w_{0}\right\|_{L^{\infty}(\Omega)}^{1-\beta}\right)^{\frac{1}{1-\beta}} .
$$

Now, from all the above lemmas, we get

Theorem 6. If the initial data satisfy Equation (3) and $\beta>1$, then the solution satisfies

$$
\lim _{t \rightarrow \infty}\|p(\cdot, t)\|_{L^{\infty}(\Omega)}+\|w(\cdot, t)\|_{L^{\infty}(\Omega)}=0 .
$$


Moreover, for any $\epsilon \in(0,1)$, there exist $t_{\epsilon}$ such that for $t>t_{\epsilon}>0$

$$
\|p(\cdot, t)-1\|_{L^{\infty}(\Omega)} \leq C(\epsilon)\left(\left\|w_{0}\right\|_{L^{\infty}(\Omega)}^{1-\beta}+\gamma(\beta-1)(1-\epsilon)\left(t-t_{\epsilon}\right)\right)^{\frac{1}{2(1-\beta)}},
$$

and

$$
\|w(\cdot, t)\|_{W^{1,2}(\Omega)} \leq C\left(\gamma(\beta-1)(1-\epsilon)\left(t-t_{\epsilon}\right)+\left\|w_{0}\right\|_{L^{\infty}(\Omega)}^{1-\beta}\right)^{\frac{1}{1-\beta}} .
$$

4.2. Asymptotical Behavior When $\beta=1$

Lemma 26. If $\beta=1$ and the initial data satisfy Equation (3), then we have

$$
\lim _{t \rightarrow \infty}\|p(\cdot, t)-1\|_{L^{\infty}(\Omega)}=0,
$$

and

$$
\lim _{t \rightarrow \infty}\|w(\cdot, t)\|_{L^{\infty}(\Omega)}=0 .
$$

Proof. Equation (91) can be checked by repeating the proof in Lemma 24. Now, we focus on the proof of Equation (92).

By solving the $w$-equation, we obtain

$$
\begin{aligned}
|w(x, t)| & \leq\left\|w_{0}\right\|_{L^{\infty}(\Omega)} e^{-\gamma \int_{0}^{t} p(s) d s}, \\
& \leq\left\|w_{0}\right\|_{L^{\infty}(\Omega)} e^{\gamma \int_{0}^{t}\|p(s)-\bar{p}(s)\|_{L^{\infty}(\Omega)}-\gamma \int_{0}^{t} \bar{p}(s) d s}, \\
& \leq\left\|w_{0}\right\|_{L^{\infty}(\Omega)} e^{\frac{\gamma}{\eta} \int_{0}^{t}\|p(s)-\bar{p}(s)\|_{L^{\infty}(\Omega)}^{2}+\eta \gamma t-\int_{0}^{t} \bar{p}(s) d s} .
\end{aligned}
$$

Then, the Poincare-iWirtinger inequality and the Sobolev embedding theorem imply that

$$
\begin{aligned}
\int_{0}^{t}\|p(\cdot, s)-\bar{p}(s)\|_{L^{\infty}(\Omega)} & \leq C \int_{0}^{\infty}\|\nabla p(\cdot, s)\|_{L^{2}(\Omega)} d s, \\
& \leq \sup _{t \geq 0}\|p(\cdot, t)\|_{L^{\infty}(\Omega)} C \int_{0}^{\infty} \int_{\Omega} \frac{|\nabla p(\cdot, s)|^{2}}{p} d s .
\end{aligned}
$$

By applying the above inequality, we have

$$
\|w(\cdot, t)\|_{L^{\infty}(\Omega)} \leq\left\|w_{0}\right\|_{L^{\infty}(\Omega)} e^{\frac{\gamma C}{\eta}+\gamma \eta t-\int_{0}^{t} \bar{p}(s) d s} .
$$

Equation (48) implies that we can choose $t_{0}$ such that for $t>t_{0}$

$$
\bar{p}(t)>\frac{1}{2}
$$

Thus, Equation (93) indicates that

$$
\|w(\cdot, t)\|_{L^{\infty}(\Omega)} \leq\left\|w_{0}\right\|_{L^{\infty}(\Omega)} e^{\left(\gamma \eta-\frac{1}{2}\right) t+\frac{\gamma \mathcal{C}}{\eta}+\frac{1}{2} t_{0}} .
$$

Then, we pick $\gamma \eta-\frac{1}{2}<0$ to obtain Equation (92).

Lemma 27. For any $\epsilon \in\left(0, \min \left\{1, \gamma, \lambda, \lambda_{1}\right\}\right)$, there exists $C(\epsilon)>0$ such that

$$
\|p(\cdot, t)-\bar{p}(t)\|_{L^{\infty}(\Omega)} \leq C(\epsilon) e^{-\left(\min \left\{1, \gamma, \lambda, \lambda_{1}\right\}-\epsilon\right) t}
$$

where $\lambda_{1}$ is the first nonzero eigenvalue of $-\Delta$ with homogeneous Neumann boundary condition. 
Proof. Notice that $\lim _{t \rightarrow \infty}|| p(\cdot, t)-1 \|_{L^{2}(\Omega)}=0$ and estimate $\int_{\Omega}|\nabla w|^{2} \leq C e^{-2 \gamma(1-\epsilon) t}$ are still valid in the one-dimensional setting.

By integrating the $p$-equation over $\Omega$, we deduce

$$
\frac{d}{d t} \bar{p}(t)=\lambda \overline{p(1-p)}
$$

By applying the variation-of-constant formula to $p-\bar{p}$, we get $p(\cdot, t)-\bar{p}(t)=e^{t \Delta}(p(\cdot, 0)-\bar{p}(0))-\rho \int_{0}^{t} e^{(t-s) \Delta} \operatorname{div}(p \nabla w) d s+\lambda \int_{0}^{t} e^{(t-s) \Delta}(p(1-p)-\overline{p(1-p)}) d s$.

With the Sobolev inequality, we can obtain

$$
\begin{aligned}
\|p(\cdot, t)-\bar{p}(t)\|_{L^{\infty}(\Omega)} \leq k_{1} e^{\lambda_{1} t}|| p(\cdot, 0)-\bar{p}(0) \|_{L^{\infty}(\Omega)} & +k_{2} \int_{0}^{t}\left(1+(t-s)^{-\frac{3}{4}}\right) e^{-\lambda_{1}(t-s)}\|\nabla w\|_{L^{2}(\Omega)} d s \\
& +k_{3} \int_{0}^{t}\left(1+(t-s)^{-\frac{3}{4}}\right) e^{-\lambda_{1}(t-s)}\|p-1\|_{L^{2}(\Omega)} d s .
\end{aligned}
$$

Then, the well-known $L^{p}-L^{q}$ estimates of heat semigroup(e.g., [22]) imply that

$$
\begin{array}{r}
\|p(\cdot, t)-\bar{p}(t)\|_{L^{\infty}(\Omega)} \leq k_{1} e^{-\lambda_{1} t}|| p(\cdot, 0)-\bar{p}(0) \\
\|_{L^{\infty}(\Omega)}+k_{2}(\epsilon) \int_{0}^{t}\left(1+(t-s)^{-\frac{3}{4}}\right) e^{-\min \left\{\gamma, 1, \lambda, \lambda_{1}\right\}(1-\epsilon) t} d s \\
+k_{3}(\epsilon) \int_{0}^{t}\left(1+(t-s)^{-\frac{3}{4}}\right) e^{\min \left\{\gamma, 1, \lambda, \lambda_{1}\right\} \epsilon s-\min \left\{\gamma, 1, \lambda, \lambda_{1}\right\} t} d s
\end{array}
$$

Hence, the result of Lemmas 16 and 17 show that

$$
\|p(\cdot, t)-\bar{p}(t)\|_{L^{\infty}(\Omega)} \leq C(\epsilon) e^{-\left(\min \left\{\gamma, 1, \lambda, \lambda_{1}\right\}-\epsilon\right) t}
$$

where $\epsilon \in\left(0, \min \left\{\gamma, 1, \lambda, \lambda_{1}\right\}\right)$.

Lemma 28. For any $\epsilon \in\left(0, \min \left\{\gamma, 1, \lambda, \lambda_{1}\right\}\right)$, there exists constant $C(\epsilon)>0$ such that

$$
|\bar{p}(t)-1| \leq C(\epsilon) e^{-\left(\min \left\{\gamma, 1, \lambda, \lambda_{1}\right\}-\epsilon\right) t}
$$

Proof. By integrating the $p$-equation over spatial variable, we have

$$
\frac{d}{d t}(\bar{p}-1)=-\lambda \bar{p}(\bar{p}-1)-\frac{\lambda}{|\Omega|}\|p(\cdot, t)-\bar{p}(t)\|_{L^{2}(\Omega)}^{2} .
$$

Now, we choose some $\epsilon>0$ and $t_{\epsilon}$ such that $\bar{p}(t)>1-\epsilon$ whenever $t>t_{\epsilon}$. By applying the Gronwall inequality, we have

$$
\begin{aligned}
|\bar{p}(t)-1| & \leq\left|\bar{p}\left(t_{\epsilon}\right)-1\right| e^{-\lambda \int_{t_{\epsilon}}^{t} \bar{p}(s) d s}+\frac{\lambda}{|\Omega|} \int_{t_{\epsilon}}^{t} e^{-\lambda \int_{s}^{t} \bar{p}(u) d u}|| p(s)-\left.\bar{p}(s)\right|_{L^{2}(\Omega)} ^{2} d s \\
& \leq\left|\bar{p}\left(t_{\epsilon}\right)-1\right| e^{-\lambda(1-\epsilon)\left(t-t_{\epsilon}\right)}+\frac{\lambda}{|\Omega|} \int_{0}^{t} e^{-\lambda(1-\epsilon)(t-s)} e^{-2\left(\min \left\{\gamma, 1, \lambda, \lambda, \lambda_{1}\right\}-\epsilon\right) s} d s .
\end{aligned}
$$

For the last term on the right side of all the above inequalities, it holds that

$$
\begin{aligned}
\int_{0}^{t} e^{-\lambda(1-\epsilon)(t-s)} e^{-2\left(\min \left\{\gamma, 1, \lambda, \lambda_{1}\right\}-\epsilon\right) s} d s & \leq \int_{0}^{t} e^{-\min \left\{\gamma, 1, \lambda, \lambda_{1}\right\}(1-\epsilon)(t-s)} e^{-2\left(\min \left\{\gamma, 1, \lambda, \lambda_{1}\right\}-\epsilon\right) s} d s \\
& \leq \int_{0}^{t} e^{\left(\left(2-\min \left\{\gamma, 1, \lambda, \lambda_{1}\right\}\right) \epsilon-\min \left\{\gamma, 1, \lambda, \lambda_{1}\right\}\right) s-\min \left\{\gamma, 1, \lambda, \lambda_{1}\right\}(1-\epsilon) t} d s \\
& \leq c_{6}(\epsilon) e^{-\left(\min \left\{\gamma, 1, \lambda, \lambda_{1}\right\}-\epsilon\right) t} .
\end{aligned}
$$


Along all inequalities above, we get

$$
|\bar{p}(t)-1| \leq C(\epsilon) e^{-\left(\min \left\{\gamma, 1, \lambda, \lambda_{1}\right\}-\epsilon\right) t}
$$

where $\epsilon \in\left(0, \min \left\{\gamma, 1, \lambda, \lambda_{1}\right\}\right)$.

Lemma 29. For any $\epsilon \in\left(0, \min \left\{\gamma, 1, \lambda, \lambda_{1}\right\}\right)$, there exists $C(\epsilon)>0$ such that

$$
\|p(\cdot, t)-1\|_{L^{\infty}(\Omega)} \leq C(\epsilon) e^{-\left(\min \left\{\gamma, 1, \lambda, \lambda_{1}\right\}-\epsilon\right) t}
$$

Proof. It is observed that

$$
\|p(\cdot, t)-1\|_{L^{\infty}(\Omega)} \leq\|p(\cdot, t)-\bar{p}(t)\|_{L^{\infty}(\Omega)}+|\bar{p}(t)-1| .
$$

Then, Lemmas 27 and 28 imply Equation (102).

Lemma 30. For any $\epsilon \in(0,1)$, there exists $C(\epsilon)>0$ such that

$$
\|w(\cdot, t)\|_{W^{1,2}(\Omega)} \leq C(\epsilon) e^{-\gamma(1-\epsilon) t} .
$$

Proof. It results from Equation (92) and estimate $\int_{\Omega}|\nabla w|^{2} \leq C e^{-2 \gamma(1-\epsilon) t}$.

Now, we conclude all the above lemmas as the following result

Theorem 7. If the initial data satisfy Equation (3), then the solution

$$
\lim _{t \rightarrow \infty}\|p(\cdot, t)\|_{L^{\infty}(\Omega)}+\|w(\cdot, t)\|_{L^{\infty}(\Omega)}=0 .
$$

Moreover, for any $\epsilon \in\left(0, \min \left\{\lambda_{1}, 1, \gamma, \lambda\right\}\right)$, there exists $C(\epsilon)>0$ such that

$$
\|p(\cdot, t)\|_{L^{\infty}(\Omega)} \leq C(\epsilon) e^{-\left(\min \left\{\lambda_{1}, 1, \gamma, \lambda\right\}-\epsilon\right) t},
$$

and

$$
\|w(\cdot, t)\|_{L^{\infty}(\Omega)} \leq C(\epsilon) e^{-\gamma(1-\epsilon) t},
$$

where $\lambda_{1}$ is the first nonzero eigenvalue of $-\Delta$ in $\Omega$ with the homogeneous Neumann boundary condition.

Author Contributions: All the authors contributed to each part of this study equally. All authors have read and agreed to the published version of the manuscript.

Funding: This research received no external funding.

Conflicts of Interest: The authors declare no conflict of interest.

\section{References}

1. Anderson, A.R.; Chaplain, M.A.; Newman, E.L.; Steele, R.J.; Thompson, A.M. Mathematical modelling of tomour invasion and metastasis. Comput. Math. Methods Med. 2000, 2, 129-154.

2. Stevens, A.; Othmer, H.G. Aggregation, blowup, and collapse: The $\mathrm{ABC}^{\prime}$ s of taxis on reinforced random walks. SIAM J. Appl. Math. 1997, 57, 1044-1081. [CrossRef]

3. Levine, H.A.; Pamuk, S.; Sleeman, B.D.; Nilsen-Hamilton, M. Mathematical modeling of capillary formation and development in tumor angiogenesis: Penetration into the stroma. Bull. Math. Biol. 2001, 63, 801-863. [CrossRef] [PubMed]

4. Levine, H.A.; Sleeman, B.D.; Nilsen-Hamilton, M. A mathematical model for the roles of pericytes and macrophages in the initiation of angiogenesis. I. The role of protease inhibitors in preventing angiogenesis. Math. Biosci. 2000, 168, 77-115. [CrossRef] 
5. Paweletz, N.; Knierim, M. Tumor-related angiogenesis. Crit. Rev. Oncol. Hematol. 1989, 9, 197-242. [CrossRef]

6. Sleeman, B.D. Mathematical modelling of tumor growth and angiogenesis. In Oxygen Transport to Tissue XIX; Springer: Boston, MA, USA, 1997; pp. 671-677.

7. Corrias, L.; Perthame, B.; Zaag, H. A chemmotaxis model motivated by angiogenesis. Comptes Rendus Mathematique 2003, 336, 141-146. [CrossRef]

8. Corrias, L.; Perthame, B.; Zaag, H. Global solutions of some chemotaxis and angiogenesis system in high space dimensions. Milan J. Math. 2004, 72,1-28. [CrossRef]

9. Guarguaglini, F.R.; Natalini, R. Global existence of solutions to a nonlinear model of sulphation phenomena in calcium carbonate stones. Nonlinear Anal. RWA 2005, 6, 477-494. [CrossRef]

10. Liţcanu, G.; Morales-Rodrigo, C. Global solutions and asymptotic behavior for a parabolic degenerate coupled system arising from biology. Nonlinear Anal. TMA 2010, 72, 77-98. [CrossRef]

11. Rascle, M.; Ziti, C. Finite time blow-up in some models of chemotaxis. J. Math. Biol. 1995, 33, $388-414$. [CrossRef] [PubMed]

12. Rascle, M. On a system if nonlinear strongly coupled partial differential equations arising in biology. In Ordinary and partial Differential Equations; Springer: Berlin/Heidelberg, Germany, 1981; pp. 290-298.

13. Bournaveas, N.; Calvez, V. The one-dimensional Keller-Segel model with fractional diffusion of cells. Nonlinearity 2009, 23, 923-935. [CrossRef]

14. Burczak, J.; Granero-Belinchón, R. On a generalized doubly parabolic Keller-Segel system in one spatial dimension. Math. Model Method Appl. Sci. 2016, 26, 111-160. [CrossRef]

15. Belmonte-Beitia, J.; Woolley, T.E.; Scott, J.G.; Maini, P.K.; Gaffney, E.A. Modelling biological invasions: Individual to population sacles at interfaces. J. Theor. Biol. 2013, 334, 1-12. [CrossRef] [PubMed]

16. Engwer, C.; Hunt, A.; Surulescu, C. Effective equations for anisotropic glioma spread with proliferation: A mutiscale approach and comparisons with previous setting. Math. Med. Biol. 2015, 33, 435-459. [CrossRef] [PubMed]

17. Winkler, M. Singular structure formation in a degenerate haptotaxis model involving myopic diffusion. Journal de Mathématiques Pures et Appliquées 2018, 112, 118-169. [CrossRef]

18. Fontelos, M.A.; Friedman, A.; Hu, B. Mathematical analysis of a model for initiation of angiogenesis. SIAM J. Math. Anal. 2002, 33, 1330-1355. [CrossRef]

19. Friedman, A.; Tello, J.I. Stability of solutions of chemotaxis equations in reinforced random walks. J. Math. Anal. 2002, 272, 138-163. [CrossRef]

20. Morales-Rodrigo, C.; Tello, J.I. Global existence and asymptotic behavior of a tumor angiogenesis model with chemotaxis and haptotaxis. Math. Model Method Appl. Sci. 2014, 24, 427-464. [CrossRef]

21. Kinderlehrer, D. An Introduction to Variational Inequalities and Their Applications; Academic Press: Cambridge, MA, USA, 1980.

22. Winkler, M. Aggregation vs. global diffusive behavior in the higher-dimensional Keller-Segel model. J. Differ. Equ. 2010, 248, 2889-2905. [CrossRef]

(C) 2020 by the authors. Licensee MDPI, Basel, Switzerland. This article is an open access article distributed under the terms and conditions of the Creative Commons Attribution (CC BY) license (http://creativecommons.org/licenses/by/4.0/). 